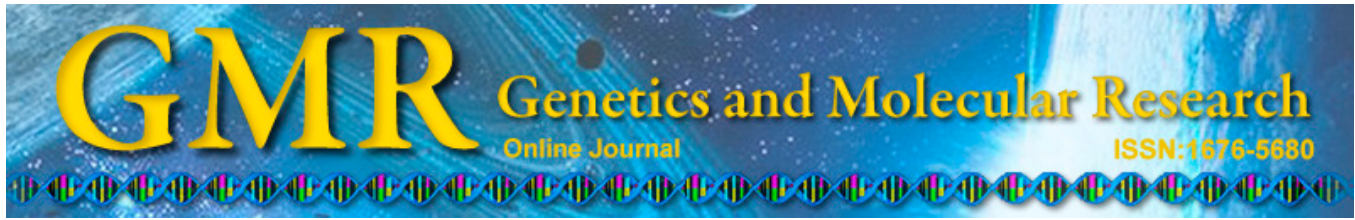

\title{
Identification of conservation units of the hynobiid salamander Pachyhynobius shangchengensis
}

\author{
L.-N. Su ${ }^{1,2 *}$, Y.-Y. Zhao ${ }^{1,3 *}$, X.-M. Wu' ${ }^{2}$ H.-F. Zhang ${ }^{2}$ and X.-C. Li ${ }^{1}$ \\ ${ }^{1}$ College of Life Sciences of Shaanxi Normal University, Xi'an, China \\ ${ }^{2}$ Shaanxi Institute of Zoology, \\ Northwest Institute of Endangered Zoological Species, Xi'an, China \\ ${ }^{3}$ Department of Cell Biology and Genetics, \\ Zunyi Medical University, Zunyi, China \\ *These authors contributed equally to this study. \\ Corresponding author: X.-C. Li \\ E-mail: xiaochen@snnu.edu.cn
}

Genet. Mol. Res. 14 (3): 9772-9778 (2015)

Received December 28, 2014

Accepted May 8, 2015

Published August 19, 2015

DOI http://dx.doi.org/10.4238/2015.August.19.10

\begin{abstract}
The evolutionary significant units (ESUs) of the salamander Pachyhynobius shangchengensis (Hynobiidae) in the Dabieshan mountains, southeastern China, were identified based on mitochondrial DNA data. We used methods for detecting cryptic species, such as the minimum spanning tree, the automatic barcode gap discovery, and the generalized mixed Yule-coalescent model; geographical partitioning was also used to identify the ESUs. A total of four ESUs were identified.
\end{abstract}

Key words: Conservation unit; Cytochrome c oxidase subunit I; Cytochrome b; Pachyhynobius shangchengensis; China 


\section{INTRODUCTION}

Ryder (1986) described conservation units as "evolutionarily significant units, ESUs", and Moritz (1994) defined management units (MUs) as "significant divergence of allele frequencies at nuclear or mitochondrial loci, regardless of the phylogenetic distinctiveness of the alleles". Genetic divergence is one of the most important criteria for the identification of ESUs (Ryder, 1986; Dizon et al., 1992; Moritz, 1994; De Guia and Saitoh, 2007). Another criterion for identifying ESUs is geographic partitioning (Dizon et al., 1992).

The salamander Pachyhynobius shangchengensis (Hynobiidae) was first described by Fei et al. (1985) in Henan Province, China, and it is endemic to the Dabieshan mountains, southeastern China. It is a rare and endangered species (Wu et al., 1994; Cai, 2001; Fei et al., 2006; Wang et al., 2009; Xiong et al., 2009; Duan et al., 2010). Based on phylogenetic studies, four MUs (Zhao et al., 2013) or three ESUs (Pan et al., 2014) have been identified. The four MUs recognized by Zhao et al. (2013) are actually four ESUs, since they were identified based on the criterion of reciprocal monophyly. The genetic criterion for the identification of an ESU is that "ESUs should be reciprocally monophyletic for mtDNA alleles, and exhibit significant divergence of allele frequencies at nuclear loci" (Moritz, 1994). ESUs are characterized by significant genetic distances (Dizon et al., 1992). Methods for detecting cryptic species, such as the minimum spanning tree constructed using TCS 1.21 (Clement et al., 2000), the automatic barcode gap discovery (ABGD) (Puillandre et al., 2012), and the generalized mixed Yule-coalescent (GMYC) model developed by Pons et al. (2006), could be used to identify ESUs. These methods may be effective at identifying ESU boundaries.

We investigated four natural populations of this species: the Jingangtai (JGT) population, the Huangbaishan (HBSH) population, the Tiangtangzhai (TTZH) population, and the Yingshan-Yuexi-Huoshan (YSHX-YXX-HSHX) population. All of the populations were geographically separated from each other (Figure 1). The present study aimed to identify the ESUs of $P$. shangchengensis using methods that can detect cryptic species, and compare the results with the reported descriptions of the ESUs for this species (Zhao et al., 2013; Pan et al., 2014). Mitochondrial cytochrome b (mtDNAcyt b) and cytochrome c oxidase subunit I (mtDNA COI) were used as molecular markers in the analysis.

\section{MATERIAL AND METHODS}

\section{Sampling and DNA sequence data}

A total of 79 P. shangchengensis individuals were collected from six locations in the Dabieshan mountains, southeastern China (Figure 1). The mtDNAcyt b (942 bp) and mtDNA COI (1011 bp) data were the same as used by Zhao et al. (2013).

\section{Data analysis}

A minimum spanning tree was built using TCS 1.21 (Clement et al., 2000) based on combined mtDNA cyt $b$ and mtDNA COI data. The cryptic species hypothesis was tested us- 


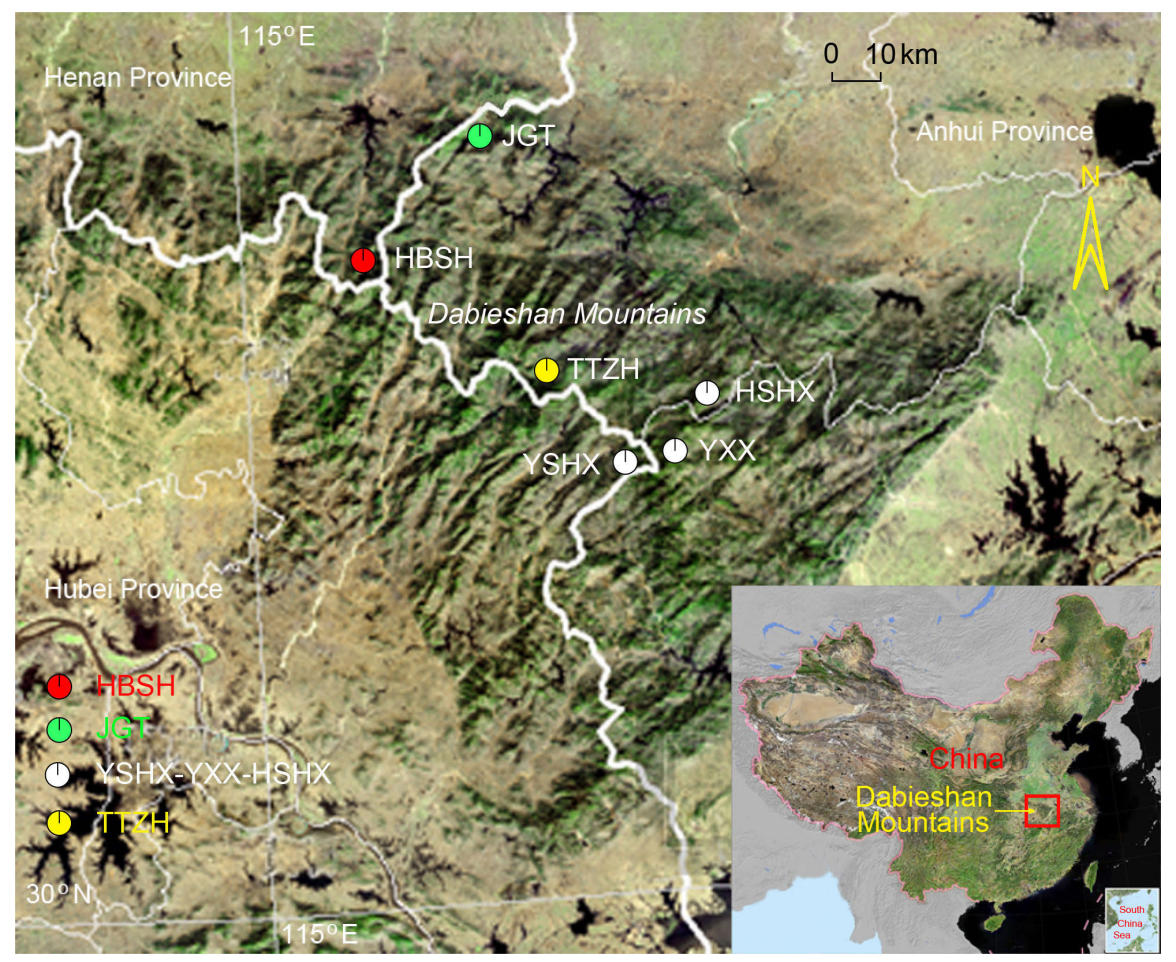

Figure 1. Geographical distribution of the four evolutionary significant units (ESUs) of Pachyhynobius shangchengensis. These four ESUs include HBSH, JGT, YSHX-YXX-HSHX, and TTZH and are represented by circles in different colors.

ing ABGD based on combined mtDNA cyt $b$ and mtDNA COI data. The ABGD web-interface, as well as a command-line program, is available at http://wwwabi.snv.jussieu.fr/public/abgd/ abgdweb.html (Puillandre et al., 2012). The prior intraspecific divergence was set to between 0.01 and 0.03 (Puillandre et al., 2012).

A GMYC model developed by Pons et al. (2006) and Monaghan et al. (2009) was used to detect the ESUs. This analysis required a rooted ultrametric tree, which was constructed in BEAST v. 1.6.1 (Drummond and Rambaut, 2007). The GMYC model has been successfully used to detect cryptic species (Fontaneto et al., 2009, 2011); therefore, we analyzed our data with the GMYC model using the R package's "splits" (species' limits by threshold statistics) (r-forge.rproject.org/projects/splits/, accessed 2011; R Development Core Team, 2008) to check for cryptic species within P. shangchengensis. We used the single-threshold method since it outperforms the multiple-threshold method (Fujisawa and Barraclough, 2013). Splits between cryptic species and major intraspecific lineages were estimated at a substitution rate of $0.64 \%$ per million years per branch (lineage), as suggested by Weisrock et al. (2001) for hynobiid salamander mitochondrial DNA. This evolutionary rate has been used previously for hynobiid mitochondrial DNA data (Matsui et al., 2007, 2008; Yoshikawa et al., 2008; Malyarchuk et al., 2010). The GMYC analysis of COI and cyt b was performed consecutively, since the GMYC procedure is based on single-locus sequence data. The geographical distances between the ESUs were calculated online at http://www.gpsvisualizer.com/calculators. 


\section{RESULTS}

The maximum parsimony network yielded four unconnected subnetworks (Figure 2). Subnetwork HBSH included all of the haplotypes from HBSH, subnetwork JGT included all of the haplotypes from JGT, subnetwork TTZH included all of the haplotypes from TTZH, and subnetwork YSHX-YXX-HSHX included all of the haplotypes from YSHX-YXX-HSHX. The ABGD analysis detected four distinct groups (Figure 3). Group 1 included all of the haplotypes from HBSH, group 2 included all of the haplotypes from HSHX, YSHX, and YXX, group 3 included all of the haplotypes from JGT, and group 4 included all of the haplotypes from TTZH.

The GMYC analysis (based on cyt $b$ ) detected four independent evolutionary entities in P. shangchengensis (Figure 4); there was a significant difference between this and the null hypothesis of one single species $(\mathrm{P}=0.007)$. Entity 1 included all of the haplotypes from HBSH, entity 2 included all of the haplotypes from JGT, entity 3 included all of the haplotypes from HSHX, YSHX, and YXX, and entity 4 included all of the haplotypes from TTZH (Figure 4). The GMYC analysis based on COI failed to give satisfactory results.

The minimum geographical distance between the ESUs was $20.5 \mathrm{~km}$ (Figure 5); therefore, the four ESUs were completely geographically isolated.

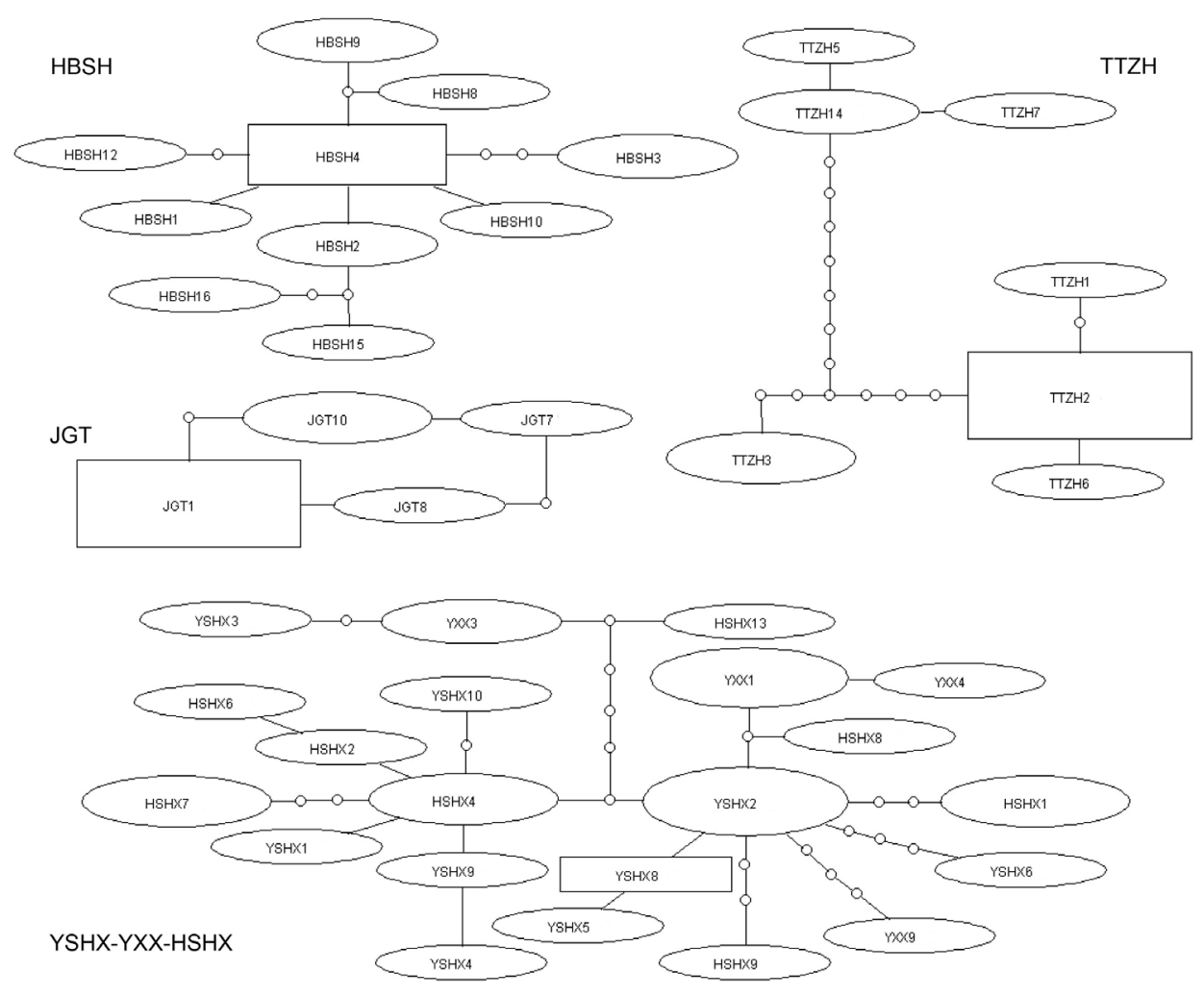

Figure 2. Minimum spanning tree of haplotypes based on the combined data. Each haplotype is represented by an ellipse or square (ancestor), with the area proportional to its frequency. 


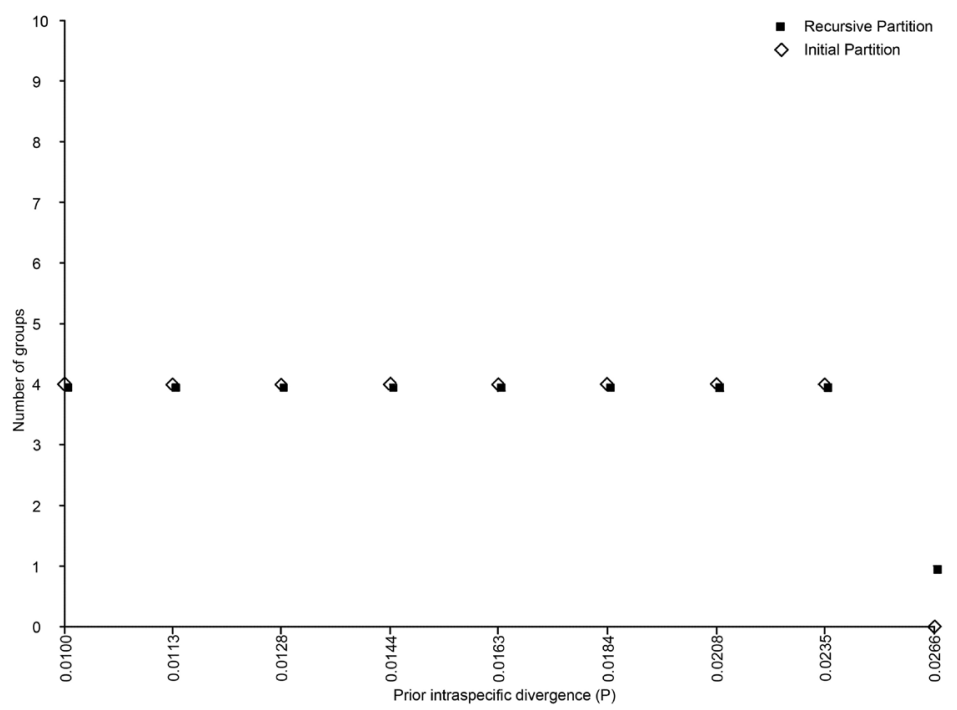

Figure 3. Analysis results of ABGD (automatic barcode gap discovery) based on the combined data.

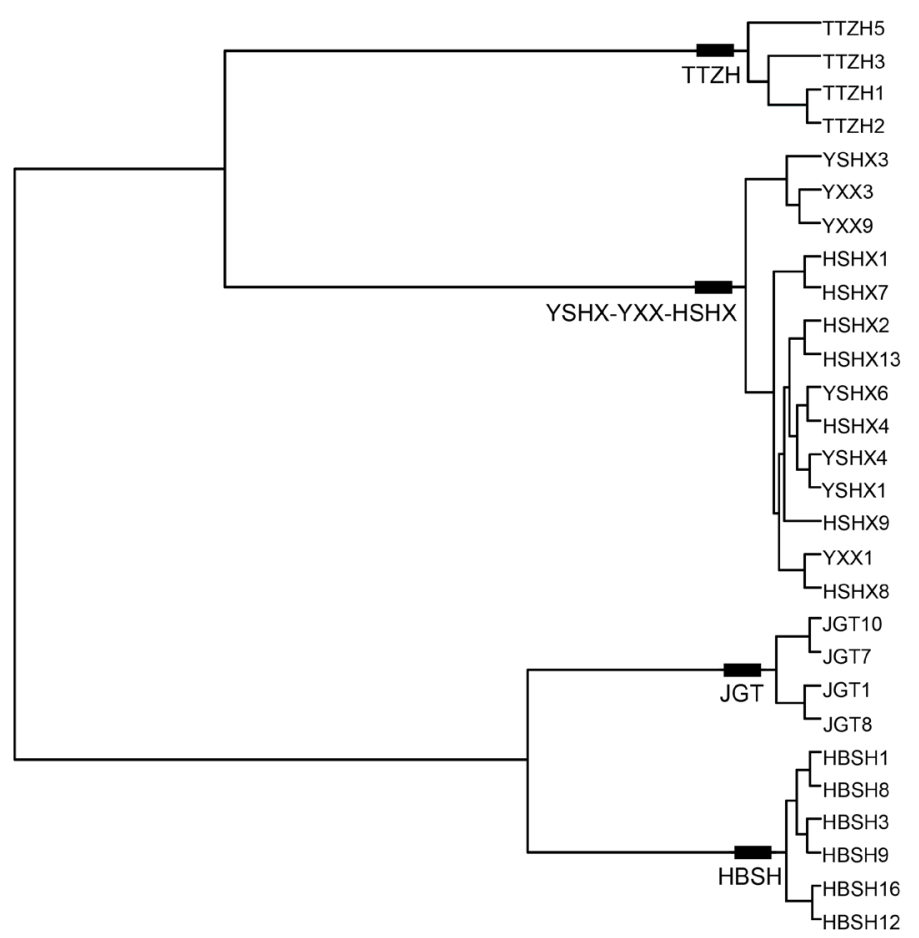

Figure 4. GMYC model analysis based on cyt $\mathrm{b}$ fragment. Number of entities $=4\left(\mathrm{P}=0.007^{* *}\right)$. Rectangles indicate groups identified by the GMYC model as independent evolutionary entities (populations). 


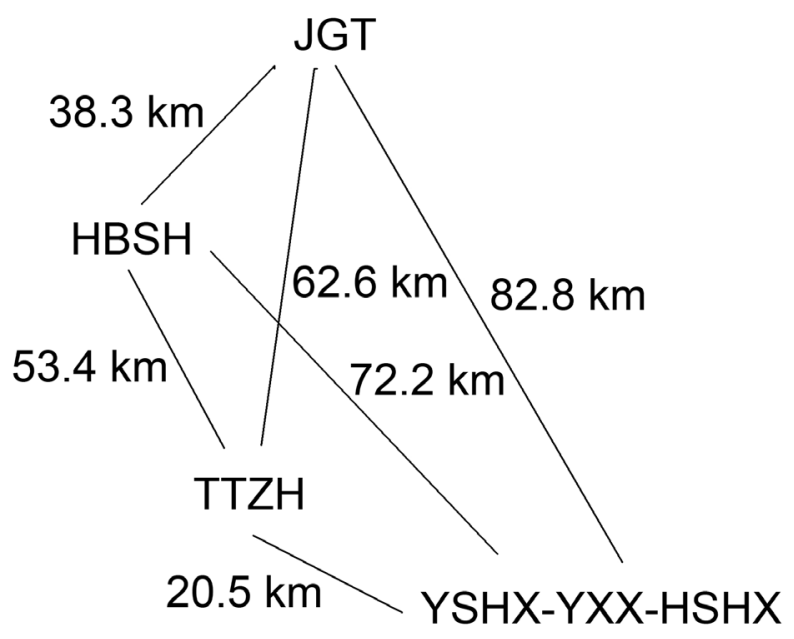

Figure 5. Geographical distances between the evolutionary significant units.

\section{DISCUSSION}

The maximum parsimony network yielded four unconnected subnetworks suggesting that there are four cryptic species in P. shangchengensis. The ABGD and GMYC analyses confirmed the existence of four cryptic species. The same lineage (subnetwork, group, or entity) in different analyses (maximum parsimony network, ABGD, and the GMYC model) was composed of the haplotypes that came from the same locality. Different cryptic speciesdetection analyses yielded the same results, so it can be concluded that there are four ESUs (HBSH, JGT, TTZH, and YSHX-YXX-HSHX) for P. shangchengensis (Figure 1). These four ESUs are geographically isolated from each other, which decreases the level of gene flow between them. Significant geographical partitioning is one of the most important criteria for ESU identification (Dizon et al., 1992).

Four ESUs were identified, which is consistent with the results of Zhao et al. (2013) but differs from the results of Pan et al. (2014). There are at least four criteria for the identification of ESUs: significant genetic differentiation, geographical isolation, ecological traits, and morphometric data (De Guia and Saitoh, 2007), and the absence of any of these criteria will yield only partial ESUs (De Guia and Saitoh, 2007). For the identification of $P$. shangchengensis ESUs, we could only satisfy the significant genetic differentiation and geographical isolation criteria, since no morphological or ecological differences between the ESUs were detected.

\section{ACKNOWLEDGMENTS}

Research supported by Project \#31071888 of the National Natural Science Foundation of China and the Science and Technology Program of Shaanxi Academy of Science (2014 K-33, 2008K-02). 


\section{REFERENCES}

Cai SY (2001). The ecological observation and resources protection of Pachyhynobius shangchengensis. J. Central China Norm. Univ. (Nat. Sci.) 35: 203-205.

Clement M, Posada D and Crandall KA (2000). TCS: a computer program to estimate gene genealogies. Mol. Ecol. 9: 1657-1660.

De Guia APO and Saitoh T (2007). The gap between the concept and definitions in the Evolutionarily Significant Unit: the need to integrate neutral genetic variation and adaptive variation. Ecol. Res. 22: 604-612.

Dizon AE, Lockyer C, Perrin WF, Demaster D, et al. (1992). Rethinking the stock concept: a phylogeographic approach. Conserv. Biol. 6: 24-36.

Drummond AJ and Rambaut A (2007). BEAST: Bayesian evolutionary analysis by sampling trees. BMC Evol. Biol. 7: 214.

Duan HS, Yang ZQ, Xu G, Zhou SL, et al. (2010). Study on the delimitation and zoogeographical regions of amphibians fauna in Hubei area. Hubei Agr. Sci. 49: 3149-3152.

Fei L, Qu WY and Wu SH (1985). Description of a new genus and species of Hynobiidae of China. Zool. Res. 6: 399-404.

Fei L, Hu SQ, Ye CY and Huang YZ (2006). Genus Pachyhynobius Fei, Qu et Wu 1983. In: Editorial Committee of Fauna Sinica, Amphibia (Vol. 1), Fauna Sinica (Chinese Academy of Sciences, eds.). Science Press, Beijing, 176-180.

Fontaneto D, Kaya M, Herniou EA and Barraclough TG (2009). Extreme levels of hidden diversity in microscopic animals (Rotifera) revealed by DNA taxonomy. Mol. Phylogenet. Evol. 53: 182-189.

Fontaneto D, Iakovenko N, Eyres I, Kaya M, et al. (2011). Cryptic diversity in the genus Adineta Hudson \& Gosse, 1886 (Rotifera: Bdelloidea: Adinetidae): a DNA taxonomy approach. Hydrobiologia 662: 27-33.

Fujisawa T and Barraclough T (2013). Delimiting species using single-locus data and the generalized mixed Yule coalescent approach: a revised method and evaluation on simulated data sets. Syst. Biol. 62: 707-724.

Malyarchuk B, Derenko M, Berman D, Perkova M, et al. (2010). Phylogeography and molecular adaptation of Siberian salamander Salamandrella keyserlingii based on mitochondrial DNA variation. Mol. Phylogenet. Evol. 56: 562-571.

Matsui M, Tominaga A, Hayashi T, Misawa Y, et al. (2007). Phylogenetic relationships and phylogeography of Hynobius tokyoensis (Amphibia: Caudata) using complete sequences of cytochrome $b$ and control region genes of mitochondrial DNA. Mol. Phylogenet. Evol. 44: 204-216.

Matsui M, Yoshikawa N, Tominaga A, Sato T, et al. (2008). Phylogenetic relationships of two Salamandrella species as revealed by mitochondrial DNA and allozyme variation (Amphibia: Caudata: Hynobiidae). Mol. Phylogenet. Evol. 48: 84-93.

Monaghan MT, Wild R, Elliot M, Fujisawa T, et al. (2009). Accelerated species inventory on Madagascar using coalescentbased models of species delineation. Syst. Biol. 58: 298-311.

Moritz C (1994). Defining 'evolutionarily significant units' for conservation. Trends Ecol. Evol. 9: 373-375.

Pan T, Wang H, Hu CC, Shi WB, et al. (2014). Range-wide phylogeography and conservation genetics of a narrowly endemic stream salamander, Pachyhynobius shangchengensis (Caudata, Hynobiidae): implications for conservation. Genet. Mol. Res. 13: 2873-2885.

Pons J, Barraclough TG, Gomez-Zurita J, Cardoso A, et al. (2006). Sequence based species delimitation for the DNA taxonomy of undescribed insects. Syst. Biol. 55: 595-609.

Puillandre N, Lambert A, Brouillet S and Achaz G (2012). ABGD, Automatic Barcode Gap Discovery for primary species delimitation. Mol. Ecol. 21: 1864-1877.

R Development Core Team (2008). R: A language and environment for statistical computing. Vienna, Austria. Available at [http://www.R-project.org].

Ryder OA (1986). Species conservation and systematics: the dilemma of the subspecies. Trends Ecol. Evol. 1: 9-10.

Wang S, Bao FY, Qi YY and Xu JX (2009). Resource distribution and habitat selection of Pachyhynobius shangchengensis in Yaoluoping Nature Reserve. Amino Acids Biotic Resour. 31: 4-7.

Weisrock DW, Macey JR, Ugurtas IH, Larson A, et al. (2001). Molecular phylogenetics and historical biogeography among salamandrids of the "true" salamander clade: rapid branching of numerous highly divergent lineages in Mertensiella luschani associated with the rise of Anatolia. Mol. Phylogenet. Evol. 18: 434-448.

Wu SH, Niu HX and Xu SL (1994). The species of Urodela Amphibia and their geographic distribution in Henan Province. J. Henan Norm. Univ. (Nat. Sci.) 22: 106-112.

Xiong JL, Sun P, Zhu WW and Liu XY (2009). A specific Hynobiidae in China: Pachyhynobius shangchengensis. Anim. Husband. Feed Sci. 30: 126-127.

Yoshikawa N, Matsui M, Nishikawa K, Kim JB, et al. (2008). Phylogenetic relationships and biogeography of the Japanese clawed salamander, Onychodactylus japonicus (Amphibia: Caudata: Hynobiidae), and its congener inferred from the mitochondrial cytochrome b gene. Mol. Phylogenet. Evol. 49: 249-259.

Zhao YY, Zhang YH and Li XC (2013). Molecular phylogeography and population genetic structure of an endangered species Pachyhynobius shangchengensis (hynobiid salamander) in a fragmented habitat of southeastern China. PLoS One 8: e78064. 